\title{
Application of Sturm's theorem to marginal stable circular orbits of a test body in spherically symmetric and static spacetimes
}

\author{
Toshiaki Ono ${ }^{1}$, Tomohito Suzuki ${ }^{1}$, NaOmasa Fushimi $^{1}$, Kei $_{\text {Yamada }}^{2}$ and Hideki Asada ${ }^{1}$ \\ 1 Faculty of Science and Technology, Hirosaki University, Hirosaki 036-8561, Japan \\ 2 Department of Physics, Kyoto University, Kyoto 606-8502, Japan
}

PACS 04.25.Nx - Post-Newtonian approximation; perturbation theory; related approximations
PACS 95.30.Sf - Relativity and gravitation

PACS 04.70.-s - Physics of black holes

\begin{abstract}
In terms of Sturm's theorem, we reexamine a marginal stable circular orbit (MSCO) such as the innermost stable circular orbit (ISCO) of a timelike geodesic in any spherically symmetric and static spacetime. MSCOs for some of exact solutions to the Einstein's equation are discussed. Strum's theorem is explicitly applied to the Kottler (often called Schwarzschild-de Sitter) spacetime. Moreover, we analyze MSCOs for a spherically symmetric, static and vacuum solution in Weyl conformal gravity.
\end{abstract}

Introduction. - In general relativity, the orbital radius has a lower bound that is called the innermost stable circular orbit (ISCO). The existence of the ISCO in the Schwarzschild solution for the Einstein's equation is fascinating [1]. Moreover, ISCOs may play key roles in astrophysics as well as in gravity theory. For instance, ISCOs are of great importance in gravitational waves astronomy [2], because ISCOs are thought to be the location a t the transition from the inspiralling phase to the merging one, especially when a compact object is orbiting around á massive black hole probably located at a galactic center. Furthermore, in high energy astrophysics, ISCOs are related to the existence for the inner edge of an accretion disk around a black hole [3]. It is thus expected that a measurement of the ISCO radius will bring us important information on the strong gravity, especially the nonlinear spacetime geometry that is beyond the solar-system tests. For near-future astrophysical tests of the no-hair theorem for black holes, therefore, it is intriguing to study the nature of the ISCOs in non-Schwarzschild spacetimes such as black holes with electric charges and/or scalar fields 4 and black holes in modified gravity theories 5 .

It has been thought that unstable circular orbits would be generally produced by strong gravity in general relativity. This is the case for the Schwarzschild spacetime. If the cosmological constant for instance is added into the Einstein's equation, however, it is not always the case. Stuchlik and Hledik 6] have pointed out that the outermost stable circular orbit (OSCO) of a test body is possi- ble in the Kottler (often called the Schwarzschild-de Sitter) spacetime [7. The ISCO and OSCO are a boundary between a stable region and an unstable one. Hereafter, we call it a marginal stable circular orbit (MSCO). Note that the wording "marginally stable" has a different meaning in the theory of dynamical systems. Hence, this paper prefers to use the wording "marginal stable circular orbits". If MSCOs are two and only two in a spacetime, one MSCO may correspond to the ISCO and the other may correspond to the OSCO. Note that the number of MSCOs might be larger than two, if such a spacetime geometry is described by a very complicated form of the metric. For this case, the smallest MSCO may be called the ISCO and the largest one may be the OSCO.

Even counting the number of MSCOs for a nonSchwarzschild metric, though it is apparently elementary, is not a straightforward task. The purpose of this brief paper is to reexamine, in terms of Strum's theorem [8], MSCOs of a timelike geodesic in any spherically symmetric and static spacetime that may have a deficit angle. MSCOs for some of exact solutions to the Einstein's equation are mentioned: Schwarzschild, Kottler (often called Schwarzschild-de Sitter) [7, Reissner-Nordström (RN) 9], Janis-Newman-Winicour (JNW) spacetimes [10, and Barriola-Vilenkin monopole with mass that is closely related to a static string 11. We illustrate how Strum's theorem is explicitly applied to the Kottler spacetime.

Moreover, we analyze a spherically symmetric, static and vacuum solution in Weyl conformal gravity, which is 
an alternative gravity model. The exterior metric to a static spherically symmetric distribution in Weyl conformal gravity was found by Mannheim and Kazanas [12]. This solution expresses a black hole for some parameter range and it is a naked singularity for another parameter region. Recently, the Weyl-gravity solution has been studied to explain rotation curves of galaxy samples without introducing a dark matter component [13] and the model may provide also a possible size of individual galaxies 14. Moreover, the gravitational lensing by this black hole model has been discussed by several groups [15, 16].

Throughout this paper, we use the unit of $G=c=1$.

Application of Strum's theorem to exact solutions for the Einstein's equation. -

Equation for a location of a MSCO. We consider spherically symmetric and static spacetimes. A general form of the line element for these spacetimes is

$$
d s^{2}=-A(r) d t^{2}+B(r) d r^{2}+C(r)\left(d \theta^{2}+\sin ^{2} \theta d \phi^{2}\right),
$$

where we assume $g_{t t} \equiv-A(r)<0, g_{r r} \equiv B(r)>0$, $g_{\theta \theta} \equiv C(r)>0$, namely the spacetime signature as ,,,-+++ . One may often choose $C(r)=r^{2}$. However, it would be more convenient to keep $C(r)$ for some cases in the literatures where $C(r) \neq r^{2}$ [10,17, because we do not need to add calculations of the coordinate transformation for reaching $C(r)=r^{2}$ for such cases.

A necessary condition for the existence of a MSCO is known as 18, 19.

$$
\frac{d}{d r}\left(\frac{1}{A(r)}\right) \frac{d^{2}}{d r^{2}}\left(\frac{1}{C(r)}\right)-\frac{d}{d r}\left(\frac{1}{C(r)}\right) \frac{d^{2}}{d r^{2}}\left(\frac{1}{A(r)}\right)=0 .
$$

This equation determines the radius of the MSCO, if the MSCO exists. Hereafter, we call Eq. (2) MSCO equation. Note that the MSCO equation does not contain a metric component $B(r)$ nor the constants of motion as $E$ and $L$.

Given a root for Eq. (2), $E^{2}$ and $L^{2}$ are expressed as

$$
\begin{aligned}
E^{2} & =-\frac{1}{\Delta} \frac{d}{d r}\left(\frac{1}{C(r)}\right), \\
L^{2} & =-\frac{1}{\Delta} \frac{d}{d r}\left(\frac{1}{A(r)}\right),
\end{aligned}
$$

where we define a determinant as

$$
\Delta \equiv\left|\begin{array}{cc}
\frac{1}{A(r)} & -\frac{1}{C(r)} \\
\frac{d}{d r}\left(\frac{1}{A(r)}\right) & -\frac{d}{d r}\left(\frac{1}{C(r)}\right)
\end{array}\right| .
$$

Any root $r$ for Eq. (2) can be substituted into Eqs. (3) and (4) in order to see whether it satisfies the sufficient condition as $0 \leq E^{2}<\infty$ and $0 \leq L^{2}<\infty$. If the sufficient condition is satisfied, this $r$ is a MSCO radius, denoted as $r_{M S C O}$. If not, the root is unphysical and it must be discarded. Note that the expressions of $E^{2}$ and $L^{2}$ in Eqs. (3) and (4) are applicable not only to MSCOs but also to any circular orbit.
MSCOs in terms of Strum's theorem . In this subsection, we apply Strum's theorem to some of exact solutions of the Einstein's equation in order to classify the number of MSCOs.

\section{Schwarzschild spacetime:}

Let us begin with the Schwarzschild spacetime as

$$
d s^{2}=-\left(1-\frac{r_{g}}{r}\right) d t^{2}+\frac{d r^{2}}{1-\frac{r_{g}}{r}}+r^{2}\left(d \theta^{2}+\sin ^{2} \theta d \phi^{2}\right),
$$

where the Schwarzschild radius is defined as $r_{g} \equiv 2 M$ for the ADM mass $M$. For this spacetime metric, Eq. (2) becomes linear as

$$
r-3 r_{g}=0,
$$

where we assume $r \neq 0$ because $r=0$ is the spacetime singularity. Hence, we obtain $r_{M S C O}=3 r_{g}$.

\section{Kottler (Schwarzschild-de Sitter) spacetime:}

Next, we consider the Kottler spacetime [7]. The line element is

$$
\begin{aligned}
d s^{2}= & -\left(1-\frac{r_{g}}{r}-\frac{\Lambda}{3} r^{2}\right) d t^{2}+\frac{d r^{2}}{1-\frac{r_{g}}{r}-\frac{\Lambda}{3} r^{2}} \\
& +r^{2}\left(d \theta^{2}+\sin ^{2} \theta d \phi^{2}\right),
\end{aligned}
$$

where $\Lambda$ denotes the cosmological constant. Note that this spacetime is not asymptotically flat. The timelike geodesics in the Kottler spacetime have been often studied by several authors 20, especially on the periastron shift, though none of them has examined the allowed region of the orbits.

For this spacetime, the MSCO equation becomes

$$
\frac{8}{3} \Lambda r^{4}-5 r_{g} \Lambda r^{3}-r_{g} r+3 r_{g}^{2}=0
$$

where we assume $r \neq 0$ and $r \neq \infty$. It is convenient to use the normalized variables in terms of the Schwarzschild radius $r_{g}$. We define $x \equiv r / r_{g}$ and $\lambda \equiv \Lambda r_{g}^{2} / 3$. The above quartic equation becomes

$$
8 \lambda x^{4}-15 \lambda x^{3}-x+3=0 .
$$

The remaining task is to investigate positive zeros for this quartic equation as a necessary condition for MSCOs. A general formula for solving a quartic equation such as Cardano's one gives a complicated form of the root. Hence, it would be preferred to use an alternative method.

Strum's theorem [8] expresses the number of distinct real roots of a polynomial $p$ located in an interval in terms of the number of changes of signs of the values of the Strum's sequence (called also the Sturm's chain) at the bounds of the interval.

Sturm's theorem: Let $p(r)$ denote a polynomial. Applying Euclid's algorithm to $p(r)$ and its derivative, Sturm's sequence is constructed as

$$
p_{0}(r) \equiv p(r)
$$




$$
\begin{aligned}
p_{1}(r) \equiv & p_{0}^{\prime}(r) \\
p_{2}(r) \equiv & p_{1}(r) q_{0}(r)-p_{0}(r), \\
p_{3}(r) \equiv & p_{2}(r) q_{1}(r)-p_{1}(r), \\
\cdots & \cdots \\
0= & p_{n}(r) q_{n-1}(r)-p_{n-1}(r),
\end{aligned}
$$

where $q_{i}(r)$ is the quotient of $p_{i}(r)$ by $p_{i+1}(r)$. Let $\mathcal{V}(a)$ denote the number of the sign changing (ignoring zeros) in the Sturm's sequence at $r=a$. Then, $\mathcal{V}(a)-\mathcal{V}(b)$ gives the number of distinct roots of $p(r)$ between $a$ and $b$, where $a<b$.

Strum's sequence for Eq. (10) in the Kottler spacetime is obtained as

$$
\begin{aligned}
f_{0}(r)= & 8 \lambda r^{4}-15 r_{g} \lambda r^{3}-r_{g}^{3} r+3 r_{g}^{4} \\
f_{1}(r)= & 32 \lambda r^{3}-45 r_{g} \lambda r^{2}-r_{g}^{3} \\
f_{2}(r)= & \frac{3}{128} r_{g}^{2}\left(225 \lambda r^{2}+32 r_{g} r-123 r_{g}^{2}\right) \\
f_{3}(r)= & \frac{128 r_{g}^{2}}{50625 \lambda} \\
& \times\left[-2(128+4725 \lambda) r+3 r_{g}(328+3375 \lambda)\right]
\end{aligned}
$$

$$
f_{4}(r)=-\frac{151875 r_{g}^{4} \lambda}{512(128+4725 \lambda)^{2}}(4-27 \lambda)(16-16875 \lambda)
$$

where $f_{4}(r)$ is a constant for the present case. Henceforth, we focus on non-Schwarzschild case $(\lambda \neq 0)$.

First, we consider the case of $\lambda>0$, for which we have

$$
\begin{aligned}
& \operatorname{sgn}\left[f_{0}(0)\right]>0, \\
& \operatorname{sgn}\left[f_{1}(0)\right]<0, \\
& \operatorname{sgn}\left[f_{2}(0)\right]<0, \\
& \operatorname{sgn}\left[f_{3}(0)\right]>0, \\
& \operatorname{sgn}\left[f_{4}(0)\right]=\operatorname{sgn}[-\lambda(4-27 \lambda)(16-16875 \lambda)],
\end{aligned}
$$

and

$$
\begin{aligned}
& \operatorname{sgn}\left[f_{0}(\infty)\right]>0 \\
& \operatorname{sgn}\left[f_{1}(\infty)\right]>0 \\
& \operatorname{sgn}\left[f_{2}(\infty)\right]>0 \\
& \operatorname{sgn}\left[f_{3}(\infty)\right]<0, \\
& \operatorname{sgn}\left[f_{4}(\infty)\right]=\operatorname{sgn}[-\lambda(4-27 \lambda)(16-16875 \lambda)]
\end{aligned}
$$

where sgn denotes the sign function.

(1) If $16 / 16875<\lambda<4 / 27$, the number of the sign changing is $\mathcal{V}(0)=2$ and $\mathcal{V}(\infty)=2$, so that there cannot exist any positive root. (2) If $0<\lambda<16 / 16875$ or $\lambda>$ $4 / 27$, the sign changing number is $\mathcal{V}(0)=3$ and $\mathcal{V}(\infty)=$ 1 , which lead to two positive roots. (2a) If $\lambda \geq 4 / 27$, however, $L^{2} \leq 0$, which means no MSCO. (2b) On the other hand, both $E^{2}>0$ and $L^{2}>0$ are satisfied by $0<\lambda<16 / 16875$, which means two MSCOs.
Next, we consider $\lambda<0$. Then, we have

$$
\begin{aligned}
& \operatorname{sgn}\left[f_{0}(0)\right]>0, \\
& \operatorname{sgn}\left[f_{1}(0)\right]<0, \\
& \operatorname{sgn}\left[f_{2}(0)\right]<0, \\
& \operatorname{sgn}\left[f_{3}(0)\right]=\operatorname{sgn}[-(328+3375 \lambda)], \\
& \operatorname{sgn}\left[f_{4}(0)\right]>0,
\end{aligned}
$$

and

$$
\begin{aligned}
& \operatorname{sgn}\left[f_{0}(\infty)\right]<0, \\
& \operatorname{sgn}\left[f_{1}(\infty)\right]<0, \\
& \operatorname{sgn}\left[f_{2}(\infty)\right]<0, \\
& \operatorname{sgn}\left[f_{3}(\infty)\right]=\operatorname{sgn}[128+4725 \lambda], \\
& \operatorname{sgn}\left[f_{4}(\infty)\right]>0 .
\end{aligned}
$$

Regardless of $\operatorname{sgn}\left[f_{3}(0)\right]$ and $\operatorname{sgn}\left[f_{3}(\infty)\right]$, we find $\mathcal{V}(0)=$ 2 and $\mathcal{V}(\infty)=1$, which lead to a single positive root. One can also see that it satisfies $E^{2}>0$ and $L^{2}>0$. If $\lambda<0$, then, there always exists a single MSCO corresponding to the ISCO.

Strum's theorem (as the necessary condition) and the positive $E^{2}$ and $L^{2}$ (as the sufficient condition) tell that there are four cases:

Case 1: $\lambda=0$. There is the single positive zero corresponding to the ISCO. This is in agreement with the Schwarzschild case.

Case 2: $0<\lambda<16 / 16875$. We have two positive zeros, namely two MSCOs, where one is corresponding to the ISCO and the other is the OSCO.

Case 3: $16 / 16875 \leq \lambda$. There is no MSCO (after the ISCO and the OSCO merge at $\lambda=16 / 16875)$. This implies that every circular orbit becomes unstable for this case. This number 16/16875 was found by Stuchlik and Hledik [6], where this is expressed as $y=12 / 15^{4}$ in Eq. (24) of their paper.

Case 4: $\lambda<0$ (anti-de Sitter case). There is only the single MSCO.

Other examples in general relativity:

Let us briefly mention MSCOs in Reissner-Nordström (RN) 9], Janis-Newman-Winicour (JNW) spacetimes [10, and Barriola-Vilenkin monopole with mass [11. MSCO equations are cubic, quadratic and linear, respectively, though at first glance the JNW case appears to lead to a higher order polynomial [17. Therefore, Strum's theorem is not needed for these cases.

Spherically symmetric, static and vacuum solution in Weyl conformal gravity:

Finally, we study a spherically symmetric, static and vacuum solution in Weyl conformal gravity [12 as an alternative gravity model. The exterior spacetime metric in vacuum regions was found by Mannheim and Kazanas 12 as

$$
\begin{aligned}
d s^{2} & =-A(r) d t^{2}+\frac{1}{A(r)} d r^{2}+r^{2}\left(d \theta^{2}+\sin ^{2} \theta d \phi^{2}\right) \\
A(r) & =1-3 \beta \gamma-\frac{\beta(2-3 \beta \gamma)}{r}+\gamma r-k r^{2}
\end{aligned}
$$


where $\beta, \gamma$ and $k$ are the integration constants to the vacuum equation in Weyl conformal gravity 12 . The $k$ parameter may play the same role as the cosmological constant in the Kottler spacetime.

It is more convenient to rearrange this original form of the metric into another one [16] as

$$
\begin{aligned}
d s^{2} & =-A(r) d t^{2}+\frac{1}{A(r)} d r^{2}+r^{2}\left(d \theta^{2}+\sin ^{2} \theta d \phi^{2}\right) \\
A(r) & =\alpha-\frac{2 m}{r}+\gamma r-k r^{2}
\end{aligned}
$$

where we defined $\alpha \equiv \sqrt{1-6 m \gamma}$, and $m \equiv \beta(2-3 \beta \gamma) / 2$. The negative mass case prohibits a circular orbit because of its repulsive force. This paper thus focuses on $m>0$ to study MSCOs.

In this paper, both cases of $\gamma \geq 0$ and $\gamma<0$ are considered. When we wish to discuss a possible explanation of galactic rotation curves in Weyl gravity, $\gamma>0$ is preferred [12,13. On the other hand, $\gamma$ parameter cannot be constrained by galactic rotation curves, if one takes the standard cosmological point of view that galactic rotation curves can be explained by introducing a dark matter component. In this stance, therefore, the sign of $\gamma$ parameter can be uncertain at present.

Let us begin with the most general case $(k \neq 0$ and $\gamma \neq 0)$. Straightforward calculations show that MSCO equation becomes quintic in $r$, where the fifth-order term is due to a coupling between $k$ and $\gamma$ parameters. The quintic equation is not algebraically tractable. Moreover, roughly speaking, $r^{2}$ terms that may play a role at cosmological distances are negligible at smaller scales such as galactic and stellar sizes compared with the linear terms. In this paper, therefore, we focus on $k=0$ and $\gamma \neq 0$ in order to neglect the $r^{2}$ terms in the metric. This case in Weyl conformal gravity may be used to discuss a modification to a stellar-mass black hole model, because $k$ can be effective only at cosmological distances as mentioned above. See also the metric for Kottler solution.

Interestingly, Eq. (22) with $k=0$ and $\gamma \neq 0$ accidentally coincides with an exact solution of Einstein equation with the quintessential matter surrounding a black hole (often called Kiselev black hole [21]), if the parameter in Kiselev black hole can be adjusted suitably $\left(3 w_{n}+1=-1\right)$. Note that the parameters of Weyl-gravity black holes and Kiselev ones have very different physical origins: The parameters in Weyl-gravity black holes are the integration constants coming from a higher derivative theory, while those in Kiselev black holes are due to the equation of state for a dark energy component, more precisely the potential form that the quintessence field obeys.

According to Eqs. (3) and (4), $E^{2}>0$ and $L^{2}>0$ for this spacetime metric are

$$
\gamma r^{2}+2 \sqrt{1-6 m} r-6 m>0
$$

and

$$
\frac{\gamma r^{2}+2 m}{\gamma r^{2}+2 \sqrt{1-6 m} r-6 m}>0
$$

respectively.

If $\gamma>0, A(r)=0$ gives the black horizon radius as

$$
r_{H}=\frac{\sqrt{1+2 m \gamma}-\sqrt{1-6 m \gamma}}{2 \gamma}
$$

If $\gamma<0, A(r)=0$ gives the black horizon radius as

$$
\begin{aligned}
r_{\text {Hin }} & =-\frac{\sqrt{1-6 m \gamma}+\sqrt{1+2 m \gamma}}{2 \gamma}, \\
r_{\text {Hout }} & =-\frac{\sqrt{1-6 m \gamma}-\sqrt{1+2 m \gamma}}{2 \gamma},
\end{aligned}
$$

where the former and the latter correspond to the inner horizon and the outer one, respectively. Therefore, the present metric is a black hole solution if and only if

$$
-\frac{1}{2 m}<\gamma<\frac{1}{6 m}
$$

The MSCO equation for $k=0$ and $\gamma \neq 0$ becomes

$$
\begin{aligned}
& \gamma^{2} r^{4}+3 \gamma \sqrt{1-6 m \gamma} r^{3}-12 m \gamma r^{2} \\
& +2 m \sqrt{1-6 m \gamma} r-12 m^{2}=0
\end{aligned}
$$

Let us define

$$
\begin{aligned}
\bar{\gamma} & \equiv 2 m \gamma, \\
\bar{r} & \equiv \frac{r}{2 m},
\end{aligned}
$$

so that the Sturm's sequences can be in a simpler form as

$$
\begin{aligned}
f_{0}(r)= & -\bar{\gamma}^{2} \bar{r}^{4}-3 \bar{\gamma} \sqrt{1-3 \bar{\gamma}} \bar{r}^{3}+6 \bar{\gamma} \bar{r}^{2} \\
& -\sqrt{1-3 \bar{\gamma}} \bar{r}+3, \\
f_{1}(r)= & -4 \bar{\gamma}^{2} \bar{r}^{3}-9 \sqrt{1-3 \bar{\gamma}} \bar{\gamma} \bar{r}^{2}+12 \bar{\gamma} \bar{r} \\
& -\sqrt{1-3 \bar{\gamma}} \\
f_{2}(r)= & -\frac{3}{16}(9-11 \bar{\gamma})^{2}+3 \sqrt{1-3 \bar{\gamma}} \bar{r} \\
& -\frac{3}{16 \bar{\gamma}}(1+13 \bar{\gamma}), \\
f_{3}(r)= & \frac{32 \bar{\gamma}}{(9-11 \bar{\gamma})^{2}}\left(9-78 \bar{\gamma}+25 \bar{\gamma}^{2}\right) \bar{r} \\
& -\frac{64 \sqrt{1-3 \bar{\gamma}} \bar{\gamma}}{(9-11 \bar{\gamma})^{2}}(19-9 \bar{\gamma}), \\
f_{4}(r)= & \frac{3(9-11 \bar{\gamma})^{2}}{16 \bar{\gamma}(3-25 \bar{\gamma})^{2}(-3+\bar{\gamma})^{2}} \\
& \times(1+\bar{\gamma})\left(1+90 \bar{\gamma}-23 \bar{\gamma}^{2}\right),
\end{aligned}
$$

where $f_{4}(r)$ is a constant. Eq. (28) is rewritten as

$$
-1<\bar{\gamma}<\frac{1}{3}
$$

Case 1: $\bar{\gamma} \geq 0$. The sign of the Sturm's sequence for this case is

$$
\begin{aligned}
& \operatorname{sgn}\left[f_{0}(0)\right]>0, \\
& \operatorname{sgn}\left[f_{1}(0)\right]<0, \\
& \operatorname{sgn}\left[f_{2}(0)\right]<0, \\
& \operatorname{sgn}\left[f_{3}(0)\right]<0, \\
& \operatorname{sgn}\left[f_{4}(0)\right]=\operatorname{sgn}\left[1+90 \bar{\gamma}-23 \bar{\gamma}^{2}\right],
\end{aligned}
$$


and

$$
\begin{aligned}
\operatorname{sgn}\left[f_{0}(\infty)\right] & <0 \\
\operatorname{sgn}\left[f_{1}(\infty)\right] & <0 \\
\operatorname{sgn}\left[f_{2}(\infty)\right] & =\operatorname{sgn}[-(9-11 \bar{\gamma})] \\
\operatorname{sgn}\left[f_{3}(\infty)\right] & =\operatorname{sgn}\left[9-78 \bar{\gamma}+25 \bar{\gamma}^{2}\right] \\
\operatorname{sgn}\left[f_{4}(\infty)\right] & =\operatorname{sgn}\left[1+90 \bar{\gamma}-23 \bar{\gamma}^{2}\right]
\end{aligned}
$$

There is a constraint as $\bar{\gamma}<1 / 3$, because there exists the $\sqrt{1-3 \bar{\gamma}}$ term in the metric. Hence, we can show

$$
\begin{aligned}
\operatorname{sgn}\left[f_{2}(\infty)\right] & <0 \\
\operatorname{sgn}\left[f_{4}(0)\right] & =\operatorname{sgn}\left[f_{4}(\infty)\right] \\
& >0
\end{aligned}
$$

Regardless of $\operatorname{sgn}\left[f_{3}(\infty)\right]$, hence, $\mathcal{V}(0)=2$ and $\mathcal{V}(\infty)=$ 1. Therefore, there exists only the single positive root. Moreover, it satisfies $E^{2}>0$ and $L^{2}>0$. Hence, there is the only one MSCO, namely ISCO.

Case 2: $\bar{\gamma}<0$. This case is rather tricky. Non-trivial calculations are needed as shown below. The sign of the Sturm's sequence for this case is

$$
\begin{aligned}
\operatorname{sgn}\left[f_{0}(0)\right] & >0 \\
\operatorname{sgn}\left[f_{1}(0)\right] & <0 \\
\operatorname{sgn}\left[f_{2}(0)\right] & =\operatorname{sgn}[1+13 \bar{\gamma}] \\
\operatorname{sgn}\left[f_{3}(0)\right] & >0 \\
\operatorname{sgn}\left[f_{4}(0)\right] & =\operatorname{sgn}\left[-(1+\bar{\gamma})\left(1+90 \bar{\gamma}-23 \bar{\gamma}^{2}\right)\right]
\end{aligned}
$$

and

$$
\begin{aligned}
& \operatorname{sgn}\left[f_{0}(\infty)\right]<0 \\
& \operatorname{sgn}\left[f_{1}(\infty)\right]<0 \\
& \operatorname{sgn}\left[f_{2}(\infty)\right]<0 \\
& \operatorname{sgn}\left[f_{3}(\infty)\right]=\operatorname{sgn}\left[-\left(9-78 \bar{\gamma}+25 \bar{\gamma}^{2}\right)\right] \\
& \operatorname{sgn}\left[f_{4}(\infty)\right]=\operatorname{sgn}\left[-(1+\bar{\gamma})\left(1+90 \bar{\gamma}-23 \bar{\gamma}^{2}\right)\right]
\end{aligned}
$$

For $-1<\bar{\gamma}<0$, one can show that

$$
\begin{array}{r}
\operatorname{sgn}[-(1+\bar{\gamma})]<0, \\
\operatorname{sgn}\left[f_{3}(\infty)\right]<0 .
\end{array}
$$

Regardless of the sign of $f_{2}(0)$, therefore, the number of the positive roots changes at

$$
\bar{\gamma}=\frac{45-32 \sqrt{2}}{23}
$$

As a result, there are three types for $-1<\bar{\gamma}<0$ : $(2-1)$ If $(45-32 \sqrt{2}) / 23<\bar{\gamma}<0$, there are three positive zeros. However, one of them is located outside the outer horizon, while the others are placed between the two horizons and they satisfy both $E^{2}>0$ and $L^{2}>0$. Namely, there are two MSCOs.

$(2-2)$ If $\bar{\gamma}=(45-32 \sqrt{2}) / 23$, the ISCO and the OSCO merge.
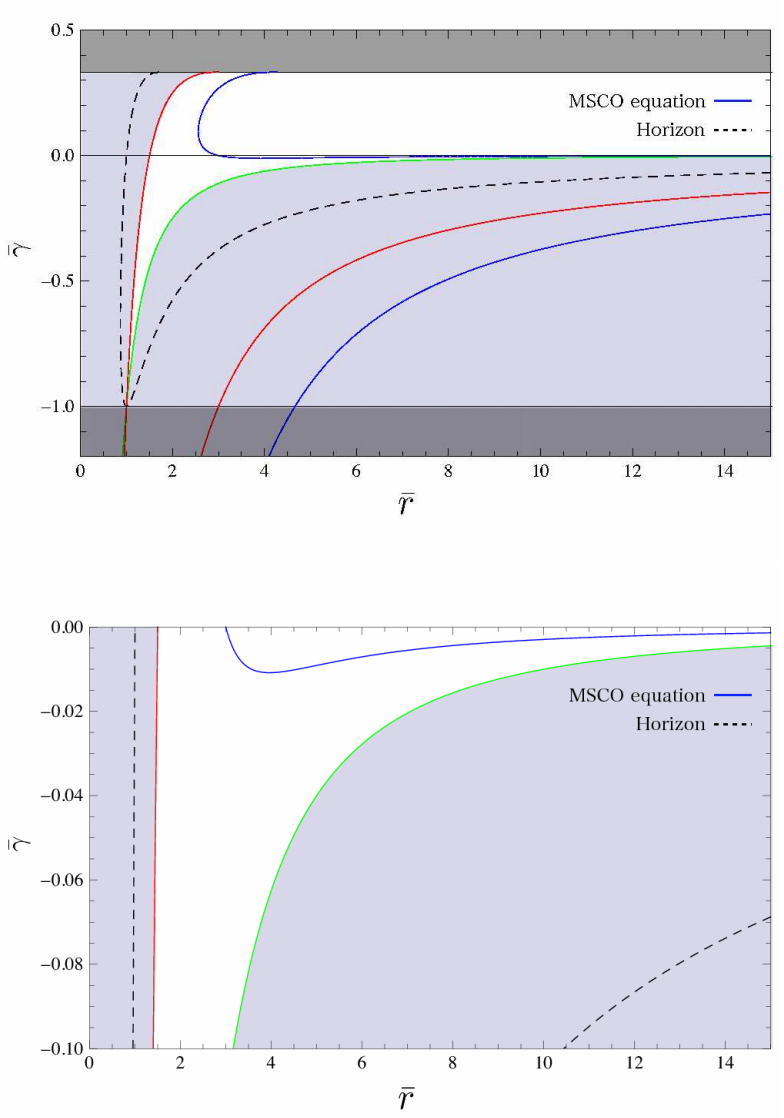

Fig. 1: $\bar{\gamma}$-dependence of the MSCO radius. The horizontal axis denotes the MSCO radius $r_{M S C O}$ as a root for the MSCO equation. The vertical axis denotes $\bar{\gamma}$ parameter. The shaded parts denote prohibited regions where $E^{2}<0, L^{2}<0, \bar{\gamma}<-1$ or $\bar{\gamma}>1 / 3$. The dashed curve in the shaded regions denotes the horizon location for a given $\bar{\gamma}$ value. Top: $\bar{\gamma} \in[-1.2,0.5]$. Bottom: $\bar{\gamma} \in[-0.1,0]$ for a zoom-up figure of the Case 2.

(2-3) If $-1<\bar{\gamma}<(45-32 \sqrt{2}) / 23$, the MSCO equation has one positive root. However, it does not satisfy $E^{2}>0$.

Bringing the Case 1 (corresponding to $\gamma>0$ ) and the Case 2 (corresponding to $\gamma<0$ ) together, Figure 1 shows the dependence of the MSCO radius on $\bar{\gamma}$.

Conclusion. - We reexamined, in terms of Strum's theorem, MSCOs of a timelike geodesic in any spherically symmetric and static spacetime that may have a deficit angle. MSCOs for some of exact solutions to the Einstein's equation were mentioned: Schwarzschild, Kottler (often called Schwarzschild-de Sitter) 7, Reissner-Nordström (RN) 9], Janis-Newman-Winicour (JNW) spacetimes [10, and Barriola-Vilenkin monopole with mass [11. We illustrated how Strum's theorem is explicitly applied to the Kottler case. This suggests that Strum's theorem is widely applicable for classifying MSCOs for some spacetime, when the MSCO equation under study is equivalent to a polynomial. 
Moreover, we analyzed MSCOs for a spherically symmetric, static and vacuum black hole solution in Weyl conformal gravity, where we neglected the square terms by assuming $k=0$. MSCO equation becomes quintic for a more general black hole solution in Weyl gravity with nonzero $k$ parameter. Numerical investigations are needed to classify MSCOs in this quintic case. It is left as a future work.

We wish to thank L. Rezzolla, T. Futamase, S. Yoshida, T. Kitamura, and Y. Sendouda for useful discussions. This work was supported in part by JSPS Grant-in-Aid for JSPS Fellows, No. 24108 (K.Y.), and JSPS Grant-inAid for Scientific Research, No. 26400262 (H.A.) and No. 15H00772 (H.A.)

\section{REFERENCES}

[1] Landau L. D. and Lifshitz E. M., The Classical Theory of Fields (Pergamon, New York) 1962; WeINBERG S., Gravitation and Cosmology, (Wiley, New York) 1972; Misner C. W., Thorne K. S. and Wheeler J. A., Gravitation (Freeman, New York) 1973; WALD R. M., General Relativity (The University of Chicago Press) 1984; Chandrasekhar S., The Mathematical Theory of Black Holes, (Oxford University Press, New York) 1998.

[2] Blanchet L., Living Rev. Relativ., 9 (2006) 4; Isoyama S., Barack L., Dolan S. R., Le Tiec A., Nakano H., Shah A. G., Tanaka T. and Warburton N., Phys. Rev. Lett., 113 (2014) 161101.

[3] Abramowicz M. A., Jaroszynski M., Kato S., Lasota J. P., Rozanska A. and Sadowski A., Astron. Astrophys., 521 (2010) A15.

[4] Bambi C. and Freese K.,, Phys. Rev. D, 79 (2009) 043002; Johannsen T. and Psaltis D., Astrophys. J., 716 (2010) 187; Johannsen T. and Psaltis D., Astrophys. J., 718 (2010) 446; Broderick A. E., JohANNSEN T., LoeB A. and Psaltis D., Astrophys. J., 784 (2014) 7.

[5] Kanti P., Mavromatos N. E., Rizos J., Tamvakis K. and Winstanley E., Phys. Rev. D, 54 (1996) 5049; Kanti P., Mavromatos N. E., Rizos J., Tamvakis K. and Winstanley E., Phys. Rev. D, 57 (1998) 6255; Yunes N. and Pretorius F., Phys. Rev. D, 79 (2009) 084043; Barausse E., Jacobson T. and Sotiriou T. P., Phys. Rev. D, 83 (2011) 124043; Konoplya R. A. and Zhidenko A., Rev. Mod. Phys., 83 (2011) 793; YAgI K., Yunes N. and Tanaka T., Phys. Rev. D, 86 (2012) 044037.

[6] Stuchlik Z. and Hledik S., Phys. Rev. D, 60 (1999) 044006.

[7] Kottler F., Ann. Phys. (Leipzig), 361 (1918) 401.

[8] B. L. van der Waerden, Algebra I (Springer, 1966)

[9] Reissner H. , Ann. Phys. (Berlin), 50 (1916) 106: Nordström G., Proc. K. Ned. Akad. Wetensch., 20 (1918) 1238.

[10] Janis A. I., Newman E. T. and Winicour J., Phys. Rev. Lett., 20 (1968) 878.
[11] Barriola M. and Vilenkin A., Phys. Rev. Lett., 63 (1989) 341.

[12] Mannheim P. D. and Kazanas D., Astrophys. J., 342 (1989) 635.

[13] Mannheim P. D. and OBrien J. G., Phys. Rev. Lett., 106 (2011) 121101

[14] Nandi K. K. and Bhadra A., Phys. Rev. Lett., 109 (2012) 079001.

[15] Sultana J. and Kazanas D., Phys. Rev. D, 81 (2010) 127502.

[16] Cattani C., Scalio M., Laserra E., Bochicchio I and Nandi K. K., Phys. Rev. D, 87 (2013) 047503.

[17] Chowdhury A. N., Patil M., Malafarina D. and Joshi P. S., Phys. Rev. D, 85 (2012) 104031.

[18] Rezzolla L. and Zhidenko A., Phys. Rev. D, 90 (2014) 084009.

[19] Barausse E., Jacobson T. and Sotiriou T. P., Phys. Rev. D, 83 (2011) 124043.

[20] Islam J. N., Phys. Lett. A, 97 (1983) 239; Cardona J. F. and Tejeiro J. M., Astrophys. J., 493 (1998) 52; Kerr A. W., Hauck J. C. and Mashhoon B., Classical Quantum Gravity, 20 (2003) 2727; Kagramanova V., Kunz J. and Lämmerzahl C., Phys. Lett. B, 634 (2006) 465; Jetzer P. and Sereno M., Phys. Rev. D, 73 (2006) 044015.

[21] Kiselev V. V., Class. Quant. Grav., 20 (2003) 1187. 